\title{
Fatty Emulsions Go Green
}

\section{Researchers create a stable dispersion of droplets that contains a high fat concentration using only unrefined, plant-derived materials.}

\section{By Katherine Wright}

$\mathrm{M}$ aking an emulsion-a mixture of two immiscible liquids in which one hosts droplets of the other-traditionally requires adding to the mixture a plethora of highly purified materials to stabilize the drops. These materials help increase the shelf-life of the emulsion, which without them might last just a few minutes. Speaking at the recent virtual Food Physics 2022 conference, Delphine Huc-Mathis of AgroParisTech, France, presented results that turn that idea on its head. She and her colleagues created highly stable emulsions from oil and water using only unrefined powdered orange pulp, called pomace, a byproduct of the juice-making industry. She says that these emulsions could find use in a wide range of products from foods to cosmetics.

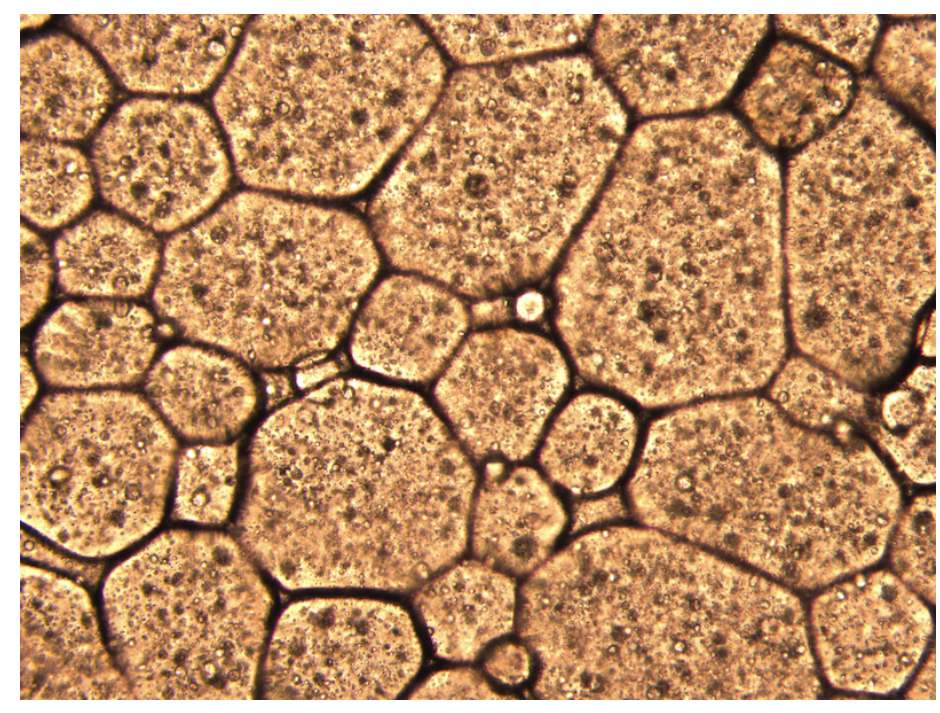

The emulsion made by Huc-Mathis and colleagues has a structure that resembles that of a foam, with large drops of oil surrounded by rivers of water.

Credit: D. Huc-Mathis/AgroParisTech

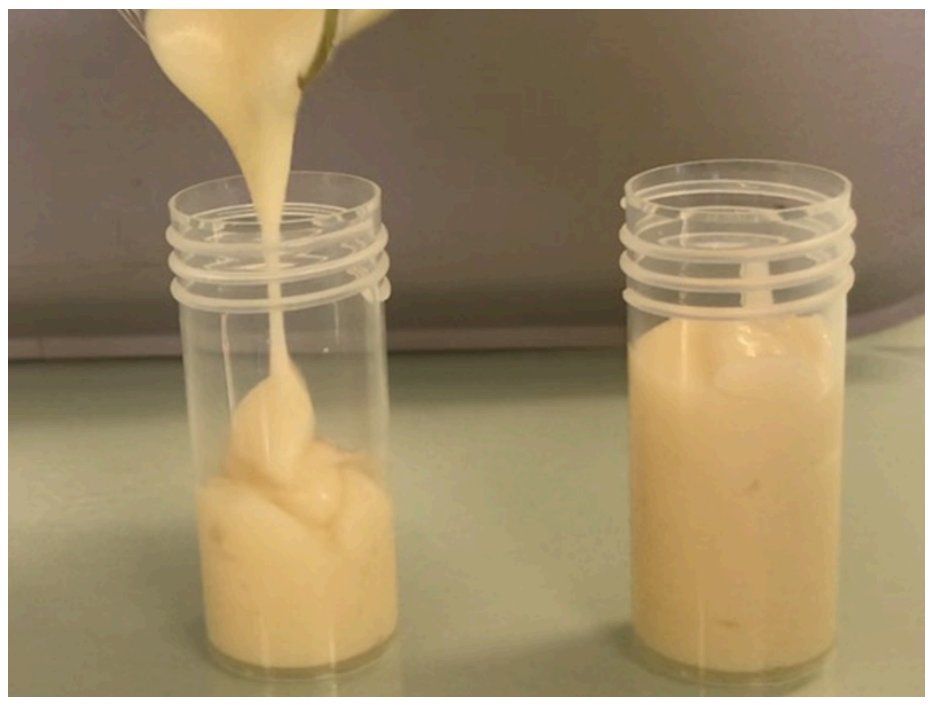

HIPPEs are known for their stability and behave like gels when poured.

Credit: D. Huc-Mathis/AgroParisTech

In a series of experiments, Huc-Mathis and her colleagues focused on making a specific type of emulsion known as a high internal phase Pickering emulsion, or HIPPE for short. Known for its stability and its solid-like properties, this emulsion contains more than $74 \%$ oil. The oil is dispersed as drops in a water phase and is generally stabilized using some surfactant material, such as nanometer-sized polymer spheres with carefully selected chemical modifications. "These materials are highly refined," Huc-Mathis says. "I wanted to see if I could make a HIPPE with an unpurified, unmodified, highly heterogenous material."

Huc-Mathis and her colleagues first mixed sunflower oil with an orange pomace powder obtained from an industrial partner. Then they added the water. Each mixing step lasted precisely 
two minutes and was done at room temperature using a standard rotor-stator mixing device that spins at 10,000 rpm. The team didn't use any heat treatment or apply any additional chemicals, Huc-Mathis says.

By varying the oil-to-water ratio, the group found that they could successfully generate an emulsion with a volume fraction of oil of $80 \%$, bringing it into the class of materials that they set out to achieve. The droplets in this emulsion remained stable both at room temperature and at elevated temperatures for at least three months, retaining their size, shape, and oil content. The final product also kept its initial strength and color-indicating that it had not been influenced by chemical reactions or by the growth of microorganisms.

The emulsion has "a very simple formulation," Huc-Mathis says. The orange pomace requires no refining, no purifying, and no modifying, and you can make it quickly with equipment that is already standard in most laboratory or industrial settings," she says. "It should be easy to scale up the process and make larger quantities." Huc-Mathis notes, however, that the formulation does have downsides: only a very narrow zone of oil and powder volume fractions successfully creates a stable emulsion. "If you add too much oil or too much powder you can have bad surprises," she says. "The emulsion just falls apart."

Huc-Mathis and her team have several ideas for the origin of the narrow recipe window, including the heterogeneity of the stabilizing orange pomace powder and its comparatively large grains compared with other emulsion-stabilizing agents. The powder particles take up "real space" compared with their nanosized synthetic counterparts, she says, and the team is beginning further studies to confirm these details. They also plan to investigate adding air to the emulsion to change its texture, something that may be of interest to the food industry.

"Our proof of concept was a success," Huc-Mathis says. "But the system is highly sensitive to formulation. Now we need to understand better why."

Katherine Wright is the Deputy Editor of Physics. 